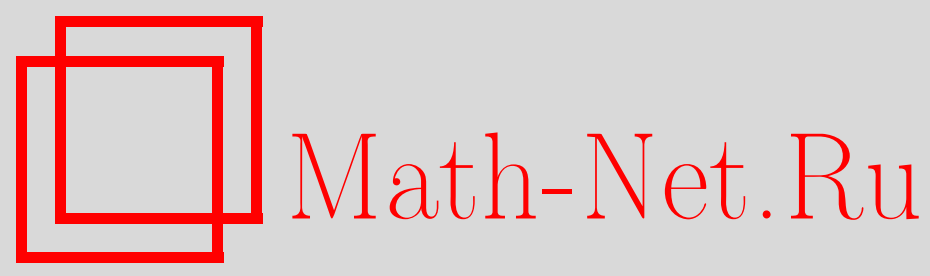

О. И. Махмудов, И. Э. Ниезов, Регуляризация решения задачи Коши для системы теории упругости в бесконечной области, Матем. заметки, 2000, том 68, выпуск 4, 548-553

DOI: https://doi.org/10.4213/mzm974

Использование Общероссийского математического портала Math-Net.Ru подразумевает, что вы прочитали и согласны с пользовательским соглашением http://www.mathnet.ru/rus/agreement

Параметры загрузки:

IP : 54.198 .187 .58

26 апреля 2023 г., $17: 13: 02$

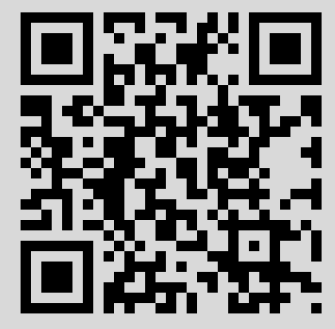




\title{
РЕГУЛЯРИЗАЦИЯ РЕШЕНИЯ ЗАДАЧИ КОШИ ДЛЯ СИСТЕМЫ ТЕОРИИ УПРУГОСТИ В БЕСКОНЕЧНОЙ ОБЛАСТИ
}

\author{
О.И. Махмудов, И. Э. Ниезов
}

\begin{abstract}
Построена матрица Карлемана задачи Коши для уравнения Гельмольца в неограниченной области $\mathbb{R}^{3}$ с кусочно-гладкой границей.

Библиография: 15 названий.
\end{abstract}

Пусть $x=\left(x_{1}, x_{2}, x_{3}\right), y=\left(y_{1}, y_{2}, y_{3}\right)$ - точки трехмерного евклидова пространства $E^{3}$ и упругая среда $D$ есть неограниченная односвязная область в $E^{3}$ с кусочно-гладкой границей, состоящей из куска $\Sigma$ гиперплоскости $y_{3}=0$ и гладкой поверхности $S$, лежащей в полупространстве $y_{3}>0$, т. е. $\partial D=S \cup \Sigma$.

Пусть вектор-функция $u(y)=\left(u_{1}(y), u_{2}(y), u_{3}(y)\right)$ в области $D$ удовлетворяет системе уравнений установившихся упругих колебаний, которая в векторной форме может быть записана в виде

$$
\mu \Delta u(y)+(\lambda+\mu) \operatorname{grad} \operatorname{div} u(y)+\rho \omega^{2} u(y)=0,
$$

здесь $\Delta$ - оператор Лапласа, $\lambda, \mu$ - постоянные Ламе рассматриваемой упругой среды, $\rho$ - плотность среды, $\omega$ - частота колебания.

Постановка задачи. Известны данные Коши решения системы (1) на поверхности $S$ :

$$
\begin{cases}u(y)=f(y), & y \in S, \\ T\left(\partial_{y}, n\right) u(y)=g(y), & y \in S,\end{cases}
$$

где $T\left(\partial_{y}, n\right)$ - оператор напряжения, т. е.

$$
T\left(\partial_{y}, n\right)=\left\|\lambda n_{k} \frac{\partial}{\partial y_{j}}+\mu n_{j} \frac{\partial}{\partial y_{k}}+\mu \delta_{k j} \frac{\partial}{\partial n}\right\|_{3 \times 3},
$$

$n=\left(n_{1}, n_{2}, n_{3}\right)$ - единичный вектор нормали к поверхности $S, f=\left(f_{1} ; f_{2}, f_{3}\right), g=\left(g_{1}\right.$; $\left.g_{2}, g_{3}\right)$ - заданные непрерьвные вектор-функции на $S, \delta_{k j}$ - символ Кронекера.

Требуется определить функцию $u(y)$ в $D$, исходя из заданных $f$ и $g$.

Задача Коши для эллиптических уравнений неустойчива относительно малого изменения данных, т. е. некорректна (пример Адамара [1], [2]). Однако если сузить класс рассматриваемых решений (класс корректности по Тихонову [1]), то для этого класса задача становится корректной. Это направление в исследовании свойств решений задачи Коши для уравнения Лапласа начато в 50-х годах в работах Лаврентьева, Мергеляна и развилось в последствии Ивановым, Ярмухамедовым, Айзенбергом и др. [3]-[9]. 
Для случая $\omega=0$ рассматриваемая задача для ограниченных областей исследована в [10]-[12]. В настоящей работе строится приближенное решение задачи (1), (2), основанное на методе Карлемана.

Развивая идею Лаврентьева, Ярмухамедов построил [6], [7], функцию Карлемана задачи Коши для уравнения Лапласа и Гельмгольца.

Ранеев работах [9], [13] было доказано, что матрица Карлемана существует во всякой задаче Коши для решений эллиптических систем, если только данные Коши задаются на граничном множестве с положительной мерой. Поскольку здесь идет речь о явньх формулах, то построение матрищы Карлемана в элементарных и специальных функциях представляет значительный интерес.

В работе строится семейство функций $u\left(x, f_{\delta}, g_{\delta}\right)=u_{\sigma \delta}(x)$, зависящее от параметра $\sigma$ и выбора параметра $\sigma(\delta)$; при $\delta \rightarrow 0$ семейство $u_{\sigma} \delta(x)$ сходится в обычном смысле к решению $u(x)$ задачи $(1),(2)$. Следуя Тихонову [14], семейство функций $u_{\sigma \delta}(x)$ назовем регуляризованным решением задачи.

Следуя [2], приведем

ОПРЕДЕЛЕНИЕ 1. Матрицей Карлемана задачи $(1),(2)$ называется $(3 \times 3)$-матрища $\Pi(y, x, \omega, \sigma)$, удовлетворяющая следующим двум условиям:

1) $\Pi(y, x, \omega, \sigma)=\Gamma(y-x, \omega)+G(y, x, \omega, \sigma)$, где $\sigma$ - положительньй числовой параметр, матрица $G(y, x, \omega, \sigma)$ по переменной $y$ удовлетворяет системе (1) всюду в области $D, \Gamma(y-x, \omega)$ - матрица фундаментальных решений уравнений $(1)$;

2) $\int_{\Sigma}\left(|\Pi(y, x, \omega, \sigma)|+\left|T\left(\partial_{y}, n\right) \Pi(y, x, \omega, \sigma)\right|\right) d S_{y} \leqslant \varepsilon(\sigma)$, где $\varepsilon(\sigma) \rightarrow 0$ при $\sigma \rightarrow \infty$; здесь и далее $|\Pi|$ означает евклидову норму матрицы $\Pi=\left\|\Pi_{k j}\right\|$, т.е. $|\Pi|=$ $\left(\sum_{k, j=1}^{3} \Pi_{k j}^{2}\right)^{1 / 2}$.

ОПРЕДЕЛЕНИЕ 2. Вектор-функция $u(y)=\left(u_{1}(y), u_{2}(y), u_{3}(y)\right)$ назьвается регулярной в $D$, если $u_{j}(y) \in C^{2}(D) \cap C^{1}(\bar{D}), j=1,2,3$.

Пусть область $D \subset E^{3}$ лежит внутри слоя $0<y_{3}<h, h=\pi / q, q>0$, и ее гранища состоит из гиперплоскости $y_{3}=0$ и гладкой поверхности $S$, заданной уравнением $y_{3}=f\left(y_{1}, y_{2}\right)$ и удовлетворяющей условиям $0<f\left(y_{1}, y_{2}\right) \leqslant h,\left|\operatorname{grad} f\left(y_{1}, y_{2}\right)\right| \leqslant$ const $<\infty,\left(y_{1}, y_{2}\right) \in E^{2}$.

Обозначим через $A(D)$ пространство всех регулярных в $D$ решений системы (1), а через $A_{q}(D)$ следующее множество:

$$
A_{q}(D)=\left\{u(y) \in A(D):|u(y)|+|\operatorname{grad} u(y)| \leqslant \exp \left(o\left(e^{q} \sqrt{y_{1}^{2}+y_{2}^{2}}\right)\right), y \rightarrow \infty, q>0\right\} .
$$

С целью построения приближенного решения задачи $(1),(2)$, рассмотрим матрицу

$$
\Pi(y, x, \omega, \sigma)=\left\|\frac{\delta_{k j}}{2 \pi \mu} \Phi\left(y, x, k_{2}, \sigma\right)-\frac{1}{2 \pi \rho \omega^{2}} \frac{\partial^{2}}{\partial y_{k} \partial y_{j}}\left[\Phi\left(y, x, k_{1}, \sigma\right)-\Phi\left(y, x, k_{2}, \sigma\right)\right]\right\|_{3 \times 3},
$$

где

$$
\begin{gathered}
\Phi(y, x, \Lambda, \sigma)=\left[-4 \pi^{2}\right]^{-1} \int_{0}^{\infty} \operatorname{Im} \frac{K\left(\sigma\left(\omega-x_{3}\right)\right)}{\omega-x_{3}} \frac{\cos \Lambda u \cdot d u}{\sqrt{u^{2}+\alpha^{2}}} \\
K(\sigma \omega)=(\omega+2 h)^{-1} \exp (\sigma \omega), \quad \omega=i \sqrt{u^{2}+\alpha^{2}}+y_{3} \\
\alpha^{2}=\left(y_{1}-x_{1}\right)^{2}+\left(y_{2}-x_{2}\right)^{2}, \quad 0<x_{3}<h .
\end{gathered}
$$

Из результатов работы [6] вытекает 
Лемма 1. Функиия $\Phi(y, x, \Lambda, \sigma)$ является функцией Карлемана для уравнения Гельмгольиа, т. е. обладает следующими двумя свойствами:

$$
\text { 1) } \Phi(y, x, \Lambda, \sigma)=\frac{\exp (i \Lambda r)}{4 \pi r}+V(y, x, \Lambda, \sigma), \quad r=|x-y|
$$

əде $V(y, x, \Lambda, \sigma)$ - некоторая функиия, определенная для всех значений $y, x$ и удовлетворяющая уравнению Гельмгольиа $\Delta(\partial / \partial y) V-\Lambda^{2} V=0, y \in D$;

2) $\quad \int_{\Sigma}(|\Phi(y, x, \Lambda, \sigma)|+|\operatorname{grad} \Phi(y, x, \Lambda, \sigma)|) d S_{y} \leqslant C(\Lambda, D) \sigma \exp \left(-\sigma x_{3}\right)$.

Докажем аналогичную лемму для системы (1).

Лемма 2. Матрииа П $(y, x, \omega, \sigma)$ является матрицей Карлемана задачи (1), (2). ДокАЗАТЕЛЬСТВо. В силу равенств (3) и (5) имеем

$$
\begin{aligned}
\Pi(y, x, \omega, \sigma)= & \| \frac{\delta_{k j}}{2 \pi \mu}\left[\frac{\exp \left(i k_{2} r\right)}{4 \pi r}+V\left(y, x, k_{2}, \sigma\right)\right] \\
& -\frac{1}{2 \pi \rho \omega^{2}} \frac{\partial^{2}}{\partial y_{k} \partial y_{j}}\left\{\left[\frac{\exp \left(i k_{2} r\right)}{4 \pi r}+V\left(y, x, k_{2}, \sigma\right)\right]\right. \\
& \left.-\left[\frac{\exp \left(i k_{2} r\right)}{4 \pi r}+V\left(y, x, k_{2}, \sigma\right)\right]\right\} \| \\
= & \Gamma(y-x, \omega)+\|\left[\frac{\delta_{k j}}{2 \pi \mu} V\left(y, x, k_{2}, \sigma\right)\right] \\
& -\frac{1}{2 \pi \rho \omega^{2}} \frac{\partial^{2}}{\partial y_{k} \partial y_{j}}\left[V\left(y, x, k_{1}, \sigma\right)-V\left(Y, x, k_{2}, \sigma\right)\right] \| \\
= & \Gamma(y-x, \omega)+\left\|G_{k j}(y, x, \omega, \sigma)\right\|_{3 \times 3}=\Gamma(y-x, \omega)+G(y, x, \omega, \sigma) .
\end{aligned}
$$

Докажем, что матрица $G(y, x, \omega, \sigma)$ по переменному у удовлетворяет системе (1) всюду в области $D$.

Пусть $G^{j}(y, x, \omega, \sigma)=\left(G_{1 j}, G_{2 j}, G_{3 j}\right), j=1,2,3$. Вектор $G^{j}(y, x, \omega, \sigma)$ в каждой точке $y \in D$ удовлетворяет системе (1) при $j=1,2,3$.

В самом деле,

$$
\begin{gathered}
\mu \Delta\left(\frac{\partial}{\partial y}\right) G^{j}(y, x, \omega, \sigma)+(\lambda+\mu) \operatorname{grad} \operatorname{div} G^{j}(y, x, \omega, \sigma) \\
=\left(R_{1 j}(y, x, \omega, \sigma), R_{2 j}(y, x, \omega, \sigma), R_{3 j}(y, x, \omega, \sigma)\right)
\end{gathered}
$$

тогда

$$
\begin{aligned}
2 \pi R_{k j}(y, x, \omega, \sigma) & \\
= & \delta_{k j} \Delta\left(\frac{\partial}{\partial y}\right) V\left(y, x, k_{2}, \sigma\right) \\
& \quad-\frac{\lambda+2 \mu}{\rho \omega^{2}} \frac{\partial^{2}}{\partial y_{k} \partial y_{j}} \Delta\left(\frac{\partial}{\partial y}\right)\left[V\left(y, x, k_{1}, \sigma\right)-V\left(y, x, k_{2}, \sigma\right)\right] \\
& +\frac{\lambda+\mu}{\mu} \frac{\partial^{2}}{\partial y_{k} \partial y_{j}} V\left(y, x, k_{2}, \sigma\right)
\end{aligned}
$$




$$
\begin{aligned}
= & -\rho \omega^{2}\left\{\frac{\delta_{k j}}{\mu} V\left(y, x, k_{2}, \sigma\right)-\frac{1}{\rho \omega^{2}} \frac{\partial^{2} V\left(y, x, k_{1}, \sigma\right)}{\partial y_{k} \partial y_{j}}\right. \\
& \left.+\frac{\lambda+2 \mu}{\mu \rho \omega^{2}} \frac{\partial^{2} V\left(y, x, k_{2}, \sigma\right)}{\partial y_{k} \partial y_{j}}-\frac{\lambda+\mu}{\mu \rho \omega^{2}} \frac{\partial^{2} V\left(y, x, k_{2}, \sigma\right)}{\partial y_{k} \partial y_{j}}\right\} \\
= & -\rho \omega^{2}\left\{\frac{\delta_{k j}}{\mu} V\left(y, x, k_{2}, \sigma\right)-\frac{1}{\rho \omega^{2}} \frac{\partial^{2}}{\partial y_{k} \partial y_{j}}\left[V\left(y, x, k_{1}, \sigma\right)-V\left(y, x, k_{2}, \sigma\right)\right]\right\} .
\end{aligned}
$$

Следовательно,

$$
R_{k j}(y, x, \omega, \sigma)=-\rho \omega^{2} G_{k j}(y, x, \omega, \sigma),
$$

что и требовалось доказать.

Используя формулы (3), (4), (5) и (6), нетрудно получить неравенство

$$
\int_{\Sigma}\left(|\Pi(y, x, \omega, \sigma)|+\left|T\left(\partial_{y}, n\right) \Pi(y, x, \omega, \sigma)\right|\right) d S_{y} \leqslant C(\Lambda, D) \sigma \exp \left(-\sigma x_{3}\right),
$$

где константа $C(\Lambda, D)$ не зависит от $x$. Лемма доказана.

Предположим теперь, что функция $u(y) \in A(D)$ ограничена вместе с нормальной производной на поверхности $\Sigma$ :

$$
|u(y)|+\left|T\left(\partial_{y}, n\right) u(y)\right| \leqslant M, \quad y \in \Sigma .
$$

В этих предложениях верна формула Грина-Купрадзе [15].

$$
2 u(x)=\int_{\partial D}\left[\Pi(y, x, \omega, \sigma)\left\{T\left(\partial_{y}, n\right) u(y)\right\}-u(y)\left\{T\left(\partial_{y}, n\right) \Pi(y, x, \omega, \sigma)\right\}\right] d S_{y}, \quad x \in D .
$$

\section{Положим}

$$
2 u_{\sigma}(x)=\int_{S}\left[\Pi(y, x, \omega, \sigma)\left\{T\left(\partial_{y}, n\right) u(y)\right\}-u(y)\left\{T\left(\partial_{y}, n\right) \Pi(y, x, \omega, \sigma)\right\}\right] d S_{y}, \quad x \in D .
$$

Верна следующая

ТЕОрема 1. Пусть $u(x) \in A_{q}(D)$ удовлетворяет граничному условию (8). Тогда имеет место оценка

$$
\left|u(x)-u_{\sigma}(x)\right| \leqslant M C_{1}(x, \Lambda, D) \sigma^{3} \exp \left(-\sigma x_{3}\right), \quad x \in D,
$$

әде $C_{1}(x, \Lambda, D)=C(\lambda, \mu, \Lambda, q) \int_{\Sigma}\left|r^{-1}+r^{-2}\right| d S_{y}, r=|y-x|$.

ДокАЗАТЕЛЬСтво. Формулу (9) перепишем в виде

$$
u(x)-u_{\sigma}(x)=\frac{1}{2} \int_{\Sigma}\left[\Pi(y, x, \omega, \sigma)\left\{T\left(\partial_{y}, n\right) u(y)\right\}-u(y)\left\{T\left(\partial_{y}, n\right) \Pi(y, x, \omega, \sigma)\right\}\right] d S_{y} .
$$

Из последнего равенства и из неравенств (7) и (8) получим (10). Теорема доказана.

Приведем результат, которьй позволяет вычислить $u(y)$ приближенно, когда на $S$ вместо $u(y)$ и $T\left(\partial_{y}, n\right) u(y)$ заданы их непрерывные приближения $f_{\delta}(y)$ и $g_{\delta}(y)$ соответственно, т. е.

$$
\max _{S}\left|u(y)-f_{\delta}(y)\right|+\max _{S}\left|T\left(\partial_{y}, n\right) u(y)-g_{\delta}(y)\right|<\delta, \quad 0<\delta<1 .
$$

Функцию $u_{\sigma \delta}(x)$ определим формулой

$$
2 u_{\sigma \delta}(x)=\int_{S}\left[\Pi(y, x, \omega, \sigma) g_{\delta}(y)-f_{\delta}(y)\left\{T\left(\partial_{y}, n\right) \Pi(y, x, \omega, \sigma)\right\}\right] d S_{y}, \quad x \in D .
$$

Верна следующая 
ТЕОрема 2. Пусть $и(y) \in A_{q}(D)$ удовлетворяет граничному условию (8). Тогда выполнена оценка

$$
\left|u(x)-u_{\sigma \delta}(x)\right| \leqslant C(x, \Lambda, D) \delta^{x_{3} / h} \ln \left(\frac{M}{\delta}\right)^{3}
$$

əде $\sigma=h^{-1} \ln (M / \delta)$

$$
C(x, \Lambda, D)=\frac{2 M^{1-x_{3} / h}}{h^{2}} C(\lambda, \mu, \Lambda, q) \int_{\partial D}\left|r^{-1}+r^{-2}\right| d S_{y}, \quad r=|y-x|
$$

ДокАЗАТЕЛьСТво. Из интегральных представлений (9) и (12) имеем

$$
\begin{aligned}
& u(x)-u_{\sigma \delta}(x)=\frac{1}{2} \int_{\Sigma}\left[\Pi(y, x, \omega, \sigma)\left\{T\left(\partial_{y}, n\right) u(y)\right\}-u(y)\left\{T\left(\partial_{y}, n\right) \Pi(y, x, \omega, \sigma)\right\}\right] d S_{y} \\
& \quad+\int_{S}\left[\Pi(y, x, \omega, \sigma)\left\{T\left(\partial_{y}, n\right) u(y)-g(y)\right\}-\left(u(y)-f_{\delta}(y)\right)\left\{T\left(\partial_{y}, n\right) \Pi(y, x, \omega, \sigma)\right\}\right] d S_{y} .
\end{aligned}
$$

Тогда по теореме 1 из условия (11) и условий теоремы получим неравенство

$$
\begin{aligned}
\left|u(x)-u_{\sigma \delta}(x)\right| \leqslant & M C_{1}(x, \Lambda, D) \sigma^{3} \exp \left(-\sigma x_{3}\right)+\delta \int_{S}[|\Pi(y, x, \omega, \sigma)| \\
& \left.+\left|T\left(\partial_{y}, n\right) \Pi(y, x, \omega, \sigma)\right|\right] d S_{y} .
\end{aligned}
$$

Очевидно, что

$$
\int_{S}\left[|\Pi(y, x, \omega, \sigma)|+\left|T\left(\partial_{y}, n\right) \Pi(y, x, \omega, \sigma)\right|\right] d S_{y} \leqslant C_{2}(x, \Lambda, D) \sigma^{3} \exp \sigma\left(h-x_{3}\right) .
$$

Объединяя эти последние два неравенства и положив $\sigma=h^{-1} \ln (M / \delta)$, получим неравенство (13). Теорема доказана.

Из теорем 1 и 2 получаем

СлЕдСТВИЕ. Предельные равенства

$$
\lim _{\sigma \rightarrow \infty} u_{\sigma}(x)=u(x), \quad \lim _{\delta \rightarrow 0} u_{\sigma \delta}(x)=u(x)
$$

выполняются равномерно на каждом компакте из D.

В заключение авторы выражают признательность академику М. М. Лаврентьеву и профессору Ш.Я. Ярмухамедову за постановку задачи и обсуждения в процессе ее решения. 


\section{СПИСОК ЦИТИРОВАННОЙ ЛИТЕРАТУРЫ}

[1] Лаврентьев М. М. О некоторых некорректных задачах математической физики. Новосибирск: ВЦ СО АН СССР, 1962.

[2] Петровский И. Г. Лекции об уравнениях с частными производньми. М.: Физматгиз, 1961.

[3] Лаврентьев М. М. О задаче Коши для уравнения Лапласа // Изв. АН СССР. Сер. матем. 1956. Т. 20. №6. С. $819-842$.

[4] Лаврентьев М. М. О задаче Коши для линейных эллиптических уравнений второго порядка // Докл. АН СССР. 1957. Т. 112. № 2. С. 195-197.

[5] Мергелян С. Н. Гармоническая аппроксимация и приближенное решение задачи Коши для уравнения Лапласа // УМН. 1956. Т. 11. № 5. С. 3-26.

[6] Ярмухамедов Ш.Я. О задаче Коши для уравнения Лапласа // Докл. АН СССР. 1977. T. 235. № 2. C. 281-283.

[7] Ярмухамедов Ш. Я., Ишанкулов Т. И., Махмудов О. И. О задаче Коши для системы уравнений теории упругости в пространстве // Сиб. матем. ж. 1992. Т. 33. № 1. С. 186-190.

[8] Иванов В.К. Задача Коши для уравнения Лапласа в бесконечной полосе // Дифференц. уравнения. 1965. Т. 1. С. 131-136.

[9] Айзенберг Л.А., Тарханов Н.Н. Абстрактная формула Карлемана // Докл. АН CCCP. 1988. Т. 298. №6. C. 1292-1296.

[10] Махмудов О. И. Задача Коши для системы упругости в пространстве. Дисс. ... к.ф.-м.н. Новосибирск, 1990.

[11] Махмудов О.И.Задача Коши для системы уравнений пространственной теории упругости в перемещениях // Изв. вузов. 1994. № 1(380). С. 54-61.

[12] Махмудов О. И., Ниезов И.Э. Регуляризация решения задачи Коши для системы уравнений теории упругости в перемещениях // Сиб. матем. ж. 1998. Т. 39. № 2. С. 369-376.

[13] Тарханов Н. Н. О матрице Карлемана для эллиптических систем // Докл. АН СССР. 1985. T. 284. № 2. C. 294-297.

[14] Тихонов А.Н. О решении некорректно поставленных задач и методе регуляризации // Докл. АН СССР. 1963. Т. 151. № 3. С. 501-504.

[15] Купрадзе В. Д., Бурчуладзе Т. В., Гегелиа Т, Г. и др. Трехмерные задачи математической теории упругости и термоупругости. М.: Наука, 1976.

Самаркандский государственный университет им. А. Навои

Поступило

E-mail : info@samuni.silk.org

16.08.1999 